\title{
Wilhelm Reich e a Produção Acadêmica Brasileira entre 1979 e 2008
}

Wilhelm Reich and the Brazilian Academic production between 1979 and 2008

Wilhelm Reich y la producción académica brasileña entre 1979 y 2008
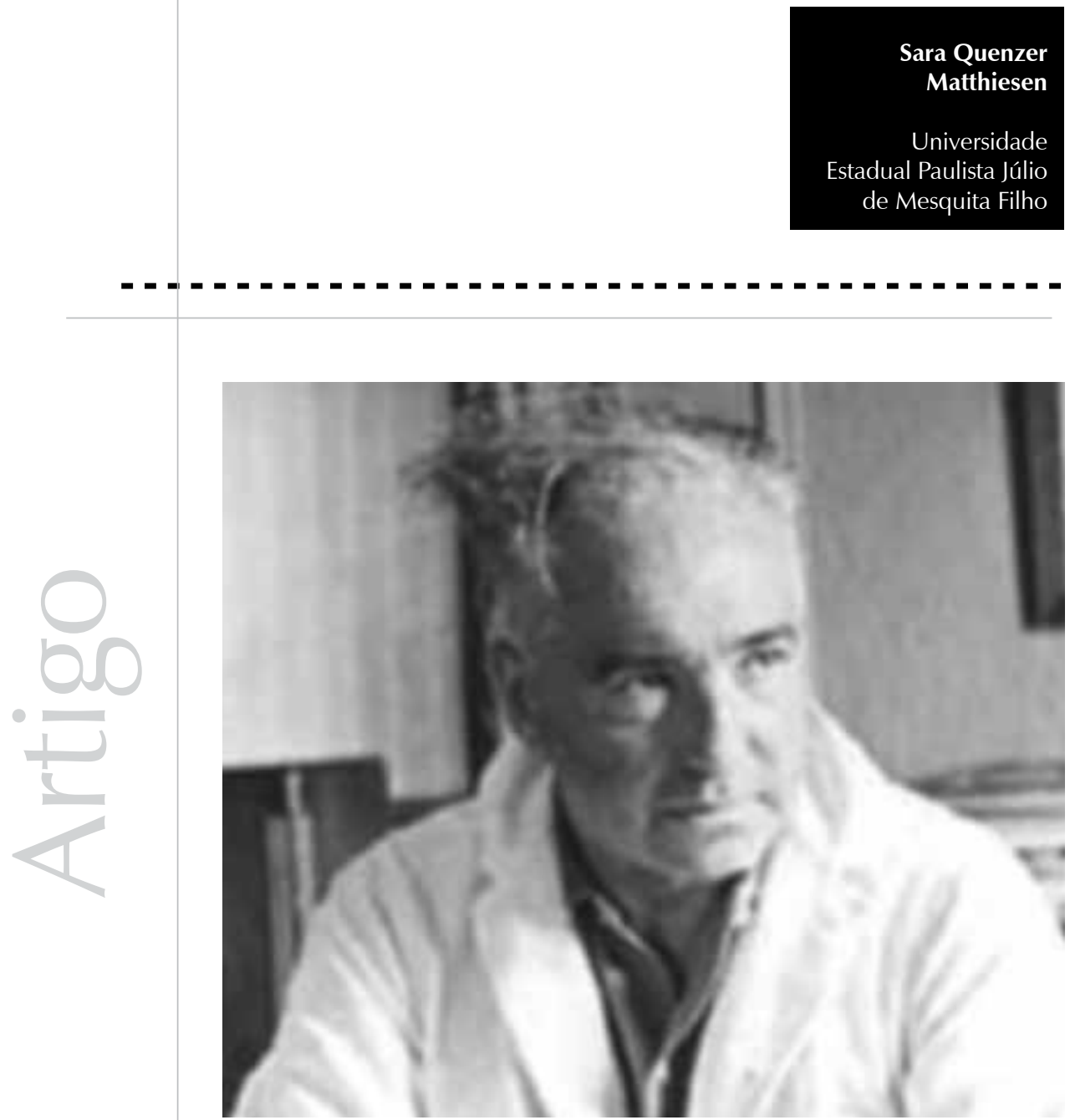
Resumo: O interesse por pesquisas relacionadas a Wilhelm Reich é notório nos últimos anos. Com o intuito de contribuir com a difusão das produções acadêmicas nesse campo, o objetivo desta pesquisa foi identificar as dissertações de mestrado e as teses de doutorado desenvolvidas em universidades brasileiras entre 1979 e 2008. Os resultados revelaram a existência de 81 produções acadêmicas, das quais 60 são dissertações de mestrado e 21, teses de doutorado. Isso nos leva a concluir que Reich tem sido objeto de interesse de pesquisadores em áreas diversas, o que evidencia que esse autor é um referencial teórico importante para a reflexão de temas contemporâneos.

Palavras-chave: Reich, Wilhelm, 1897-1957. Pesquisa científica. Pensamento reichiano.
1 Não há registro de produções reichianas nesse nível.

2 Reich expressou em seu

testamento que algumas de suas ideias só deveriam vir a público depois de 50 anos de sua morte. Sobre o assunto, ver: Reich, W. (2001). Testamento e último desejo de Wilhelm Reich. Psicologia: Teoria e Pesquisa, 17(3), 207-210 . (S.

Q. Matthiesen,

Trad.).

\begin{abstract}
The interest in research related to Wilhelm Reich's work is eminent in the recent years. Aiming to contribute to the dissemination of the academic production in this field, the goal of this research was to identify the master's degree dissertations and the doctorate thesis developed in Brazilian universities between 1979 and 2008. The results revealed the existence of 81 academic productions, 60 master's degree dissertations and 21 doctorate thesis, what leads us to conclude that Reich has been the subject of research interest in several areas, asserting him as an important theoretical reference for the reflection of contemporary issues.

Keywords: Reich, Wilhelm, 1897-1957. Scientific reseach. Reich's thought.

Resumen: El interés por pesquisas relacionadas a la Wilhelm Reich es notorio en los últimos años. Con el intuito de contribuir con la difusión de las producciones académicas en ese campo, el objetivo de esa pesquisa fue identificar las disertaciones de máster y tesis de doctorado desarrolladas en Universidades brasileñas, entre 1979 y 2008. Los resultados revelaron la existencia de 81 producciones académicas, de las cuales 60 son disertaciones de máster y 21 tesis de doctorado. Eso nos lleva a concluir que Reich ha sido objeto de interés de investigadores en áreas diversas, evidenciándolo como un referencial teórico importante para la reflexión de temas contemporáneos.
\end{abstract}

Palabras clave: Reich, Wilhelm, 1897-1957. Investigación científica. Pensamiento reichiano.

Interessada nas relações existentes entre a produção acadêmica brasileira e Wilhelm Reich, observamos (Matthiesen, 2002, 2005, 2007) ser crescente o número de dissertações de mestrado e de teses de doutorado relacionadas ao universo reichiano produzidas nos programas de pósgraduação brasileiros.

Identificado o interesse da pesquisa acadêmica sobre o tema, procuramos, neste artigo, atualizar os dados evidenciados por Matthiesen (2007) mapeando as produções acadêmicas relacionadas a Wilhelm Reich, produzidas no Brasil ao longo dos anos, de forma a favorecer o trabalho de pesquisa de outros estudiosos interessados nas possibilidades de investigação da vida e/ou obra desse autor.

\section{Procedimentos metodológicos}

Com base nos registros cadastrados no Banco de Teses da CAPES - Coordenação de Aperfeiçoamento de Pessoal de Nível Superior, que concentra pesquisas de mestrado, doutorado e profissionalizantes 1 defendidas no
Brasil entre 1987 e 2008, identificamos quais as dissertações de mestrado e as teses de doutorado relacionadas a Wilhelm Reich produzidas nos programas de pós-graduação brasileiros. Entretanto, considerando que a primeira produção acadêmica relacionada a esse autor data de 1979 (Matthiesen, 2007), delimitamos o período de análise desta pesquisa entre 1979, quando da defesa da primeira tese de doutorado relacionada ao universo reichiano, e 2008, que corresponde ao último ano de registro de produções acadêmicas no site da CAPES, até fevereiro de 2010.

Vale observar que as expressões de busca utilizadas para a localização das referências bibliográficas relacionadas ao tema foram: Wilhelm Reich, reichiana e reichiano, registrando, entre dissertações de mestrado e teses de doutorado, 81 produções acadêmicas ao longo de 29 anos (1979-2008). Isso posto, passemos à análise dos dados coletados nesta pesquisa, de modo a colaborar com a difusão dessas produções acadêmicas tão importantes para o conhecimento das ideias de Wilhelm Reich. 


\section{Resultados e discussão}

Em pesquisa realizada por Matthiesen (2007), observou-se que, entre 1979 e setembro de 2005, eram 58 as produções acadêmicas entre dissertações de mestrado (41) e teses de doutorado (17) voltadas - ainda que em níveis de aprofundamento distintos - para o universo reichiano. Entretanto, a atualização desses dados nos permite observar que, entre 1979 e dezembro de 2008, foram 81 as produções acadêmicas identificadas, das quais 60 são dissertações de mestrado e 21, teses de doutorado. Aprofundando a observação desses resultados, observamos que as pesquisas acadêmicas relacionadas a Wilhelm Reich tiveram início em 1979, intensificando-se nos anos seguintes, em especial, a partir de 2000, conforme ilustra a Tabela 1.

Tabela 1. Demonstrativo do número de dissertações de mestrado e de teses de doutorado relacionadas a Wilhelm Reich produzidas no Brasil (Modificado de: Matthiesen, 2007, p. 167)

\begin{tabular}{lccccc}
\hline Produção acadêmica & $1970-79$ & $1980-89$ & $1990-99$ & $2000-08$ & TOTAL \\
\hline Dissertação de mestrado & 0 & 3 & 14 & 43 & 60 \\
Tese de doutorado & 1 & 0 & 5 & 15 & 21 \\
TOTAL & 1 & 3 & 19 & 58 & 81 \\
\hline
\end{tabular}

Analisando a produção acadêmica relacionada ao universo reichiano (Figuras 1 e 2), observamos que houve um aumento significativo tanto em relação às dissertações de mestrado como em relação às teses de doutorado nos últimos anos. No caso da produção acadêmica do mestrado, iniciada em 1982, observamos, com base em Matthiesen (2007), que, das três dissertações defendidas entre 1980 e 1989, passou-se, na década de 1990, a 14 produções, que foram triplicadas, como demonstra a Figura 1, a partir de 2000, registrando, até dezembro de 2008, 60 dissertações de mestrado.

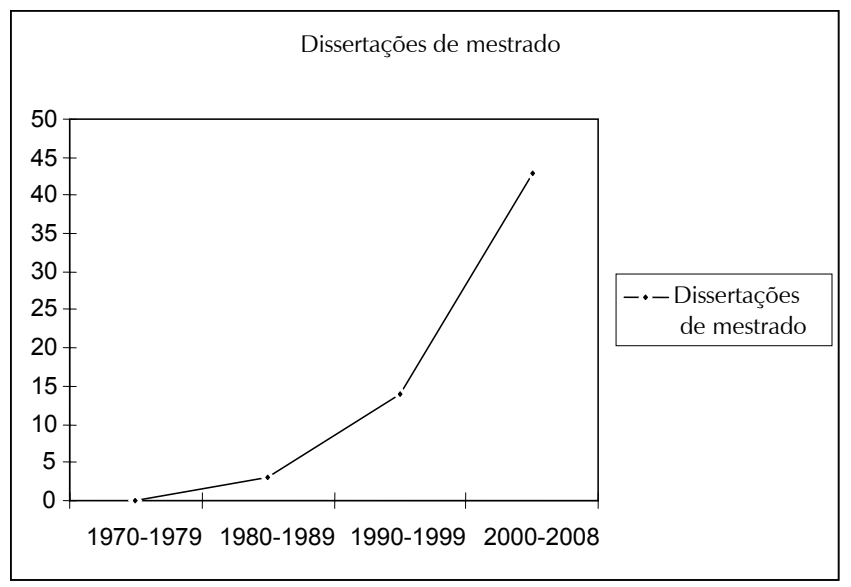

Figura 1. Dissertações de mestrado relacionadas ao universo reichiano produzidas até 2008 Fonte: Dados da pesquisa

No caso das teses de doutorado produzidas nos programas de pós-graduação brasileiros verificamos, pela Figura 2, que houve um aumento significativo na produção acadêmica, que passou de 17 trabalhos 
identificados por Matthiesen até setembro de 2005 a 21 produções até dezembro de 2008. Como vimos, a primeira - e, por muito anos, a única - tese de doutorado foi produzida na década de 70 , sendo que novos trabalhos nesse nível passaram a ser produzidos apenas na década de 90, quando identificamos 5 produções, que foram triplicadas entre 2000 e 2008, quando foram 15 as produções do total de 21 teses de doutorado defendidas.

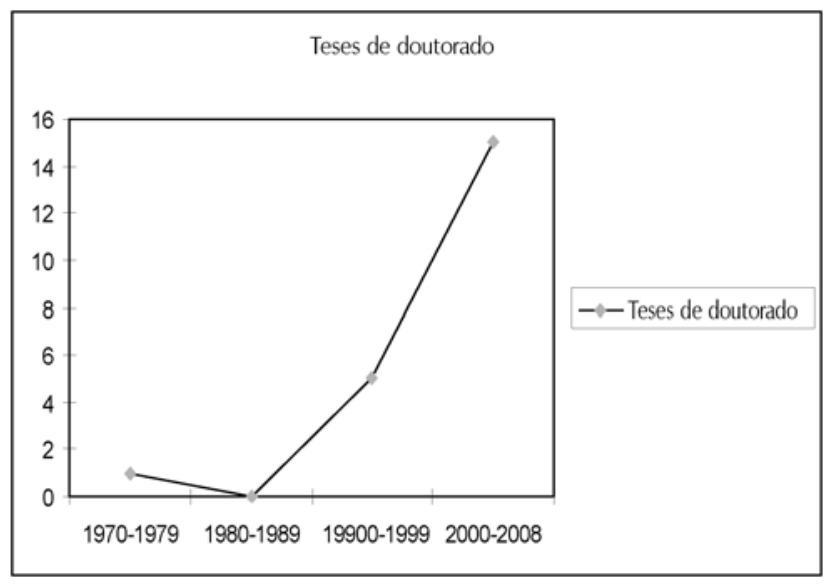

Figura 2. Teses de doutorado relacionadas ao universo reichiano produzidas até 2008 Fonte: Dados da pesquisa

Referindo-se às causas desse aumento no número de dissertações de mestrado e nas teses de doutorado, sobretudo nos últimos anos, Matthiesen (2007) menciona que a exigência por titulação - de, no mínimo, mestrado - para docência no ensino superior e a ampliação dos cursos de graduação Brasil adentro levou muitos profissionais a procurarem os cursos de pós-graduação - também crescentes no Brasil -, a fim de atenderem essa demanda. Alguns deles, já com o título de Mestre, prosseguiram estudando Reich no doutorado, certamente contribuindo para o aumento das produções nesse nível. De qualquer forma, vale observar que muitos profissionais reichianos se voltaram para a Academia a fim de registrar os resultados provenientes de anos de prática terapêutica em suas clínicas e consultórios. Assim, a "forte necessidade de registrar formalmente aquilo que se fazia empiricamente ou de sistematizar o pensamento reichiano a partir de diferentes óticas e perspectivas", como bem destacou Matthiesen (2007, p. 170), certamente promoveram uma espécie de retorno a Reich, como um referencial teórico capaz de contribuir com a reflexão de temas contemporâneos em diferentes áreas do conhecimento, como veremos adiante. Por ora, vale ainda evidenciar que a abertura e a divulgação dos Arquivos Wilhelm Reich e da permissão do Wilhelm Reich Trust Fund para a consulta do material até então preservado na Countway Library, em Boston, em função do desejo testamentário de Reich , colaborou para fazer efervescer as discussões, pesquisas, eventos e publicações na área do pensamento reichiano, sobretudo a partir de novembro de 2007, quando se completaram 50 anos da morte de Reich. Além disso, os resultados provenientes desta pesquisa evidenciam que as produções acadêmicas relacionadas ao universo reichiano não se concentram em uma só área, mas são oriundas de programas de pós-gradução de onze diferentes áreas, conforme classificação da CAPES, ilustrada pela Tabela 2. 
Tabela 2. Demonstrativo das dissertações de mestrado e teses de doutorado relacionadas ao universo reichiano por área

\begin{tabular}{lccccc}
\hline Área & Mestrado & Período & Doutorado & Período & TOTAL \\
\hline Psicologia & 19 & $1982-2008$ & 13 & $1992-2007$ & 32 \\
Educação & 23 & $1992-2008$ & 5 & $1999-2007$ & 28 \\
Artes & 4 & $1989-2005$ & - & - & 4 \\
Interdisciplinar & 3 & $2002-2008$ & 1 & 2007 & 4 \\
Ciências sociais & 3 & $2002-2008$ & 1 & 1979 & 4 \\
Comunicação & 2 & $1988-2000$ & - & - & 2 \\
Ciência Política & 2 & 1997 & - & - & 2 \\
Saúde Coletiva & 2 & $2005-2008$ & - & - & 2 \\
Educação Física & 1 & 2003 & - & - & 1 \\
Antropologia & - & - & 1 & 1991 & 1 \\
Geografia & 1 & 2008 & - & - & 1 \\
11 áreas & 60 & $1982-2008$ & 21 & $1979-2007$ & 81 \\
\hline
\end{tabular}

A análise da Tabela 2 revela que a primeira das 60 dissertações de mestrado relacionadas ao universo reichiano foi defendida na Psicologia, em 1982, área que obteve, até 2008, um total de 19 produções. Entretanto, é a área da educação que abriga o maior número de dissertações de mestrado, em um total de 23 produções entre 1992 e 2008. Na área de Artes, foram 4 as dissertações de mestrado produzidas entre 1989 e 2005, seguida pela área Interdisciplinar, com 3 dissertações de mestrado produzidas entre 2002 e 2008 e outras 3 dissertações na área de Ciências Sociais, produzidas entre 2002 e 2008; na área de Comunicação, foram 2 as dissertações entre 1988 e 2005, 2 produzidas na área de Ciência Política, em 1997 e 2007, e mais 2 na área de Saúde Coletiva, entre 2005 e 2008. Além disso, observamos a existência de 1 dissertação de mestrado em cada uma das áreas a seguir: Educação Física, em 2003, e Geografia, em 2008, totalizando as 60 dissertações de mestrado produzidas até dezembro de 2008. Em relação às teses de doutorado defendidas no Brasil relacionadas a Wilhelm Reich, observamos que a primeira - das 21 que passaram a ser produzidas apenas 12 anos depois - foi defendida junto ao Departamento de Ciências Sociais da Faculdade de Filosofia, Letras e Ciências Humanas da Universidade de São Paulo, em 1979. Depois dela, 1 foi produzida na área da Antropologia, em 1991, 13 foram produzidas na área da Psicologia, entre 1992 e 2007, 5 na de Educação, entre 1999 e 2007, e uma na área Interdisciplinar, totalizando 21 teses de doutorado investigadas até dezembro de 2008. Para que se tenha melhor ideia dessa produção, vejamos, área por área, os dados bibliográficos essenciais para a sua localização. $\mathrm{Na}$ área da Psicologia, por exemplo, observamos um total de 32 produções, sendo 19 as dissertações de mestrado e 13 as teses de doutorado defendidas sobre o assunto em programas de pós-graduação de universidades públicas (estaduais e federais) e particulares que se concentram, basicamente, no eixo Rio-São Paulo. 
Tabela 3. Produção acadêmica em Psicologia, Psicologia da saúde, Psicologia escolar e do desenvolvimento, Psicologia clínica, Psicologia social, Psicossociologia de comunidade e Ecologia social

\section{Dissertações de mestrado: 19}

Prado, P. O. B. (1982). Contribuições do pensamento e obra de Ida P. Rolf para o trabalho com postura em Psicologia. Dissertação de mestrado, Universidade de São Paulo, São Paulo, SP.

Pinheiro, M. C. T. (1992). Manifestações naturais e repressivas do comportamento sexual: um estudo exploratório na zona sul da cidade do Rio de Janeiro. Dissertação de mestrado, Universidade Federal do Rio de Janeiro, Rio de Janeiro, RJ.

Pedrini, M. M. M. (1992). Pages e a relação indivíduo-organização. Dissertação de mestrado, Universidade Metodista de São Paulo, São Paulo, SP.

Wagner, C. M. (1994). A psicanálise de Sigmund Freud e a vegetoterapia caráctero-analítica de Wilhelm Reich: continuidade ou ruptura? Dissertação de mestrado, Pontifícia Universidade Católica de São Paulo, São Paulo, SP.

Cipullo, M. A. T. (1996). O corpo da fala na fala do corpo: os lugares da palavra na bioenergética. Dissertação de mestrado, Pontifícia Universidade Católica de São Paulo, São Paulo, SP.

Brito, E. (1998). O corpo como construção imaginária: repensando as psicoterapias corporais. Dissertação de mestrado, Pontifícia Universidade Católica do Rio de Janeiro, Rio de Janeiro, RJ. Rudge, S. H. (1998). Interdisciplinaridade e psicoterapia: a construção de novos valores e perspectivas para o século XXI. Dissertação de mestrado, Universidade Federal do Rio de Janeiro, Rio de Janeiro, RJ.

Cobra, G. de O. (1999). Corpo e identidade: um estudo funcional da organização biopsíquica da identidade. Dissertação de mestrado, Pontifícia Universidade Católica do Rio de Janeiro, Rio de Janeiro, RJ.

Xavier, J. I. T. (2000). Sem corpo, sem mente: os alicerces corporais da experiência subjetiva. Dissertação de mestrado - Universidade Federal do Rio de Janeiro, Rio de Janeiro, RJ.

Cukiert, M. (2000). Uma contribuição à questão do corpo em psicanálise: Freud, Reich e Lacan. Dissertação de mestrado, Universidade de São Paulo, São Paulo, SP.

Gaiarsa, A. L. (2000). O entre: a objetividade da subjetividade. Dissertação de mestrado - Pontifícia Universidade Católica de São Paulo, São Paulo, SP.

Silva, J. R. O. e. (2001). O desenvolvimento da noção de caráter no pensamento de Reich. Dissertação de Mestrado, Universidade de São Paulo, São Paulo, SP.

Volpi, J. H. (2001). Compreendendo, por meio do relato de mães, o estresse sofrido durante a gestação e primeiros anos de vida da criança com câncer. Dissertação de mestrado, Instituto Metodista de Ensino Superior, São Bernardo do Campo, SP.

Ramalho, S. A. Psicologia de massa do fascismo: Reich e o desenvolvimento do pensamento crítico. Dissertação de mestrado, Universidade de São Paulo, São Paulo, SP.

Soares, L. G. (2003). Ritmos e conexões: dançando com Reich, Deleuze e Guattari. Dissertação de mestrado, Pontifícia Universidade Católica de São Paulo, São Paulo, SP.

Del Bianco, L. M. (2005). Alegria em uma perspectiva de saúde. Dissertação de mestrado, Pontifícia Universidade Católica de São Paulo, São Paulo, SP.

Bedani, A. (2007). Energética e epistemologia no nascimento da obra de Wilhelm Reich. Dissertação de mestrado, Universidade de São Paulo, São Paulo, SP.

Pommé, E. L. (2008). O vínculo mãe-bebê: primeiros contatos e a importância do holding. Dissertação de mestrado, Pontifícia Universidade Católica de São Paulo, São Paulo, SP. Rebello, L. L. G. R. (2008). O corpo e a clínica contemporânea: um percurso em direção à singularidade. Dissertação de mestrado, Universidade Federal Fluminense, Rio de Janeiro, RJ. 


\section{Teses de doutorado: 13}

Albertini, P. (1992). Uma contribuição para o conhecimento do pensamento de Reich: desenvolvimento histórico e formulações para a educação. Tese de doutorado, Universidade de São Paulo, São Paulo, SP.

Bellini, L. M. (1993). Afetividade e cognição: o conceito de auto-regulação como mediador da atividade humana em Wilhelm Reich e Jean Piaget. Tese de doutorado, Universidade de São Paulo, São Paulo, SP.

Câmara, M. V. A. (1999). Para além do claustro bipessoal: proposições teóricas para uma psicoterapia grupal de base reichiana. Tese de doutorado, Universidade Federal do Rio de Janeiro, Rio de Janeiro, RJ.

Lopez, L. A. (2000). Interlocuções entre a clínica fonoaudiológica e a psicologia clínica. Tese de doutorado, Pontifícia Universidade Católica de São Paulo, São Paulo, SP.

Wagner, C. M. (2000). A transferência na vegetoterapia carátero-analítica. Tese de doutorado, Pontifícia Universidade Católica de São Paulo, São Paulo.

Bezerra, D. da R. (2001). A origem e a trajetória da psicoterapia corporal: desafios da formação do psicólogo clínico em análise bioenergética. Tese de doutorado, Universidade de São Paulo, São Paulo, SP.

Cipullo, M. A. T. (2001). Entre duas abordagens: o diagnóstico psicorporal ao psicodiagnóstico fenomenológico. Tese de doutorado, Pontifícia Universidade Católica de São Paulo, São Paulo, SP. Scabaro, L. (2001). Repensando a formação psicanalítica: um novo lugar para um velho problema. Tese de doutorado, Universidade de São Paulo, São Paulo, SP.

Costa, M. de M. R. (2002). Wilhelm Reich e a bússula do pensamento funcional. Tese de doutorado, Universidade Federal do Rio de Janeiro, Rio de Janeiro, RJ.

Xavier, J. I. T. (2004). Atenção a si e psicoterapia corporal: efeitos das auto-estimulações somatossensoriais sobre a atenção e suas implicações para o corpo, as emoções e a cognição. Tese de doutorado, Universidade Federal do Rio de Janeiro, Rio de Janeiro, RJ.

Rusche, R. J. (2004). Teatro e educação somática: um estudo com presidiários em processo de criação. Tese de doutorado, Universidade de São Paulo, São Paulo, SP.

Rego, R. A. (2005). Psicanálise e biologia: uma discussão da pulsão de morte em Freud e Reich. Tese de doutorado, Universidade de São Paulo, São Paulo, SP.

Barreto, A. V. de B. (2007). A luta encarnada: corpo, poder e resistência nas obras de Foucault e Reich. Tese de doutorado, Universidade Católica de São Paulo, São Paulo, SP.

Na área da Educação, foram 28 as produções acadêmicas, sendo 23 as dissertações de mestrado e 5, as teses de doutorado defendidas sobre o assunto em programas de pós-graduação de universidades públicas (estaduais e federais) e particulares em diferentes universidades brasileiras, inclusive no interior de alguns Estados. 
Tabela 4. Produção acadêmica em Educação, História e Filosofia da Educação

\section{Dissertações de mestrado: 23}

Pereira, L. H. P. (1992). Decodificação crítica e expressão criativa: seriedade e alegria no cotidiano da sala de aula. Dissertação de mestrado, Universidade Estadual do Rio de Janeiro, Rio de Janeiro, RJ. Freitas, L. H. de. (1995). Artes cênicas na escola: o avesso do avesso do avesso. Dissertação de mestrado, Universidade do Estado do Rio de Janeiro, Rio de Janeiro, RJ.

Souza, S. M. de M. (1996). O corpo entra na escola: educação com liberdade, limite e afetoentrelaces da perspectiva reichiana e da psicomotricidade relacional. Dissertação de mestrado, Universidade Federal do Espírito Santo, Vitória, ES.

Matthiesen, S. Q. (1996). A educação do corpo e as práticas corporais alternativas: Reich, Bertherat e antiginástica. Dissertação de mestrado, Pontifícia Universidade Católica de São Paulo, São Paulo, SP.

Torresi, M. E. (1996). Era uma vez um corpo: estudo exploratório da relação mente-corpo no trabalho intelectual do professor universitário. Dissertação de mestrado, Universidade Estadual Paulista, Marília, SP.

Bilibio, L. F. S. (2002). Trágica educação sexual do erotismo: uma agonística entre Reich e Nietzsche. Dissertação de mestrado, Universidade Federal do Rio Grande do Sul, Porto Alegre, RS.

Watrin, J. P. dos S. (2002). Contribuições de Reich à luta contra a servidão voluntária. Dissertação de mestrado, Universidade Metodista de Piracicaba, Piracicaba, SP.

Moysés, M. H. F. (2003). Sensibilização e conscientização corporal do professor: influência em seus saberes e suas práticas pedagógicas. Dissertação de mestrado, Universidade Federal de Uberlândia, Uberlândia, MG.

Pagan, M. de F. F. P. B. (2003). O "holding" suficiente - práticas corporais facilitadoras no cotidiano do educador. Dissertação de mestrado, Universidade Estadual de Campinas, Campinas, SP.

Rocha, B. dos S. (2003). Brinkando na escola: o espaço escolar como criação e crescimento. Dissertação de mestrado, Universidade do Oeste Paulista, Presidente Prudente, SP.

Silva, D. A. de A. e. (2004). A ludicidade no desenvolvimento da expressão oral: uma experiência com estudantes da graduação em letras. Dissertação de mestrado, Universidade Federal da Bahia, Salvador, BA.

Bacri, A. P. R. Influências dos bloqueios corporais na aprendizagem da criança. Dissertação de mestrado, Universidade Federal de Uberlândia, Uberlândia, MG.

Pereira, V. R. T. (2005). A saúde emocional do educador: saberes necessários aos trabalhadores da educação. Dissertação de mestrado, Universidade Federal de Uberlândia, Uberlândia, MG. Sampaio, J. G. S. G. (2005). Educação e liberdade em Wilhelm Reich: reflexão sobre contribuições do pensamento reichiano para a educação. Dissertação de mestrado, Universidade Católica de Santos, Santos, SP.

Moruzzi, A. B. (2005). A escola lumiar e a questão da autonomia educativa. Dissertação de mestrado, Universidade Federal de São Carlos, São Carlos, SP.

Luz, A. M. S. (2005). Tateando em zonas (des)conhecidas e intangíveis do ser: conceitos de liberdade e sexualidade produzidas por adolescentes em situação de rua. Dissertação de mestrado, Universidade Federal do Ceará, Fortaleza, CE.

Pereira, H. H. D. (2006). Programa Saúde e Prevenção nas Escolas: políticas e gestão da educação sexual. Dissertação de mestrado, Universidade Tuiuti Paraná, Curitiba, PR.

Lelis, M. T. C. (2006). O corpo nos processos de aprendizagem: contribuições de Wilhelm Reich e Alicia Fernández. Dissertação de mestrado, Universidade Federal de Uberlândia, Uberlândia, MG. 
Sabino, S. C. (2006). O lugar do afeto na prática pedagógica na perspectiva dos professores: reflexões sobre a formação docente. Dissertação de mestrado, Universidade Católica de Petrópolis, Petrópolis, RJ.

Luiz, A. R. (2007). A história registrada no corpo das professoras: saberes docentes numa perspectiva reichiana. Dissertação de mestrado, Universidade Federal de Uberlândia, Uberlândia, MG.

Fernandes, D. M. (2008). Investigando a sexualidade de professoras: suas histórias, saberes e práticas. Dissertação de mestrado, Universidade Federal de Uberlândia, Uberlândia, MG.

Oliveira, F. I. de. (2008) Prazer! Reich! A base de uma educação livre. Dissertação de mestrado, Universidade Federal de Uberlândia, Uberlândia, MG.

Santos, J. A. S. dos (2008). Contribuição de Wilhelm Reich para a educação: visando a profilaxia da neurose. Dissertação de mestrado, Universidade Regional de Blumenau, Blumenau, SC.

\section{Teses de doutorado: 5}

Mota, M. V. S. (1999). Princípios reichianos fundamentais para a educação: base para a formação do professor. Tese de doutorado, Universidade Metodista de Piracicaba, Piracicaba, SP.

Matthiesen, S. Q. (2001). A educação em Wilhelm Reich: da psicanálise à pedagogia econômicosexual. Tese de doutorado, Universidade Estadual Paulista, Marília, SP.

Silva, E. A. da (2001). Filosofia, educação e educação sexual: matrizes filosóficas e determinações pedagógicas do pensamento de Freud, Reich e Foucault para a abordagem educacional da sexualidade humana. Tese de doutorado, Universidade Estadual de Campinas, Campinas, SP. Pereira, L. H. P. (2005). Bioexpressão: a caminho de uma educação lúdica para a formação de educadores. Tese de doutorado, Universidade Federal da Bahia, Salvador, BA.

Torresi, M. E. (2007). Relações criança-adulto no mundo contemporâneo: implicações educacionais. Tese de doutorado, Universidade Estadual Paulista, Marília, SP.

Na área de Artes, observamos um total de 4 produções, sendo todas elas dissertações de mestrado, também defendidas em programas de pós-graduação do eixo Rio-São Paulo, sem que haja quaisquer títulos de teses de doutorado vinculados ao universo reichiano.

Tabela 5. Produção acadêmica em Artes e Música

\section{Dissertações de mestrado: 4}

Azevedo, S. M. de. (1989). O papel do trabalho corporal na interpretação do ator. Dissertação de mestrado, Universidade de São Paulo, São Paulo, SP.

Monteiro, A. B. (1996). O trabalho com o corpo: uma experiência corporal. Dissertação de mestrado, Conservatório Brasileiro de Música, Rio de Janeiro, RJ.

Garcia, J. G. S. (2004). O ator vivo: uma abordagem reichiana para a arte do ator. Dissertação de mestrado, Universidade de São Paulo, São Paulo, SP.

Cabral, I. (2005). O teatro veloz: técnicas e procedimentos para um intérprete contemporâneo. Dissertação de mestrado, Universidade de São Paulo, São Paulo, SP.

Na área interdisciplinar, observamos a produção de 3 dissertações de mestrado, sendo todas do Rio de Janeiro, e 1 tese de doutorado, de Curitiba. 
Tabela 6. Produção acadêmica na área interdisciplinar

\section{Dissertações de mestrado: 3}

Santos, F. R. C. (2002). A potência orgástica e sua relação com a gravidez e o parto. Dissertação de mestrado, Universidade Gama Filho, Rio de Janeiro, RJ.

Maluf Jr., N. J. (2005). Sistêmica organísmica versus isomorfismo mente-cérebro. Dissertação de mestrado, Universidade Federal do Rio de Janeiro, Rio de Janeiro, RJ.

Rodrigues, J. L. F. (2008). A relação entre o corpo e a mente nos escritos de Freud, Lacan e Reich: do fenômeno psicossomático à unidade funcional soma-psyché. Dissertação de mestrado, Universidade Federal do Rio de Janeiro, Rio de Janeiro, RJ.

\section{Tese de doutorado: 1}

Volpi, J. H. (2007). Fundamentos epistemológicos em direção a uma ecopsicologia. Tese de doutorado, Universidade Federal do Paraná, Curitiba, PR.

$\mathrm{Na}$ área de ciências sociais, foram 3 as dissertações de mestrado e 1 tese de doutorado defendidas sobre o assunto em programas de pós-graduação de universidades públicas (estaduais e federais) e particulares.

Tabela 7. Produção acadêmica em ciências sociais e Sociologia

\section{Dissertações de mestrado: 3}

Freitas, L. E. B. de. (2002). Biosíntese e complexidade. Dissertação de mestrado, Universidade do Rio Grande do Norte, Natal, RN.

Oliveira, I. S. de. (2004). Reich - um percurso bastante singular: incursões na antropologia. Dissertação de mestrado, Pontifícia Universidade Católica de São Paulo, SP.

Silva, P. C. da. (2008). Wilhem Reich: uma leitura hermenêutica do corpo como cogito. Dissertação de mestrado, Universidade Estadual Paulista, Araraquara, SP.

\section{Tese de doutorado: 1}

Rouanet, S. P. (1979). Imaginário e poder. Tese de doutorado, Universidade de São Paulo, São Paulo, SP.

Na área de Comunicação, observamos um total de 2 produções, sendo ambas dissertações de mestrado também defendidas em programas de pós-graduação do eixo Rio-São Paulo, sem que haja quaisquer títulos de teses de doutorado vinculados ao universo reichiano. 
Tabela 8. Produção acadêmica em Comunicação, Ciências da Comunicação, Comunicação e Mercado

\section{Dissertações de mestrado: 2}

Tonon, P. de T. S. (1988). Treinamento psicofísico, uma abordagem orgânica. Dissertação de mestrado, Universidade de São Paulo, São Paulo, SP.

Polito, R. (2000). A influência da emoção do orador no processo de conquista dos ouvintes. Dissertação de mestrado, Faculdade Cásper Líbero, São Paulo, SP.

Na área da Ciência Política, observamos a produção de 2 dissertações de mestrado, ambas de universidades públicas do eixo Rio-São Paulo.

Tabela 9. Produção acadêmica em Ciência Política

\section{Dissertações de mestrado: 2}

Barreto, A. V. B. (1997). Em busca de Eros: a "democracia natural do trabalho" e a relação entre poder e afetividade no pensamento de Wilhelm Reich. Dissertação de mestrado, Universidade Estadual de Campinas, Campinas, SP.

Carvalho, A. A. de. (2007). Poder e liberdade em Wilhelm Reich: irracionalidade, poder e bio-poder. Dissertação de mestrado, Universidade Federal do Rio de Janeiro, Rio de Janeiro, RJ.

$\mathrm{Na}$ área de saúde coletiva, são duas as dissertações de mestrado defendidas em universidades públicas e nenhuma tese de doutorado, como mostra a Tabela 10.

Tabela 10. Produção acadêmica nas áreas de saúde coletiva e saúde pública

\section{Dissertações de mestrado: 2}

Souza, M. D. P. (2005). O grupo de movimento e o bem-estar subjetivo na velhice: um estudo de caso com idosos de Castelo, ES. Dissertação de mestrado, Universidade de São Paulo, São Paulo, SP.

Castel, P. V. (2008). O afeto: uma unidade psicossomática (Freud, Reich e Winnicott). Dissertação de mestrado, Universidade do Estado do Rio de Janeiro, Rio de Janeiro, RJ.

Na área da Educação Física, observamos, na Tabela 11, apenas uma dissertação de mestrado, defendida em uma universidade pública (federal) de Santa Catarina e nenhuma tese de doutorado. 
Tabela 11. Produção acadêmica na área de Educação Física

\section{Dissertações de mestrado: 1}

Bichara, V. E. A. (2003). O movimento humano numa visão reichiana: repensando a educação física escolar. Dissertação de mestrado, Universidade Federal de Florianópolis, Florianópolis, SC.

$\mathrm{Na}$ área de Antropologia, observamos apenas uma tese de doutorado, em Antropologia social, na verdade, a segunda tese de doutorado produzida no Brasil relacionada a Wilhelm Reich.

Tabela 12. Produção acadêmica em Antropologia social

\section{Tese de doutorado: 1}

Russo, J. A. (1991). O corpo contra a palavra: as terapias corporais no campo psicológico dos anos 80. Tese de doutorado, Museu Nacional do Rio de Janeiro, Rio de Janeiro, RJ.

Outra área que merece destaque é a de Geografia, que, em 2008, inaugurou, com uma dissertação de mestrado, um novo campo para a exploração da obra de Wilhem Reich, como demonstra a Tabela 13.

Tabela 13. Produção acadêmica na área de Geografia

\section{Dissertações de mestrado: 1}

Machado, M. P. (2008). A percepção ambiental dos moradores da comunidade do Passo da Pátria, em Natal/RN. Dissertação de mestrado, Universidade Federal do Rio Grande do Norte, Natal, RN.

\section{Considerações finais}

Ao término desta pesquisa, verificamos que Reich, que também fez parte do meio acadêmico, em especial, quando de sua experiência na New School for Social Research, em Nova York, onde proferiu cursos e conferências sobre Psicologia médica e técnicas psicoterapêuticas entre 1940 e 1941 (Matthiesen, 2005), hoje parece evidenciar-se como um referencial teórico passível de investigação em diferentes áreas do conhecimento, afinal, foi ele próprio que realçou essa capacidade de investigação multidisciplinar ao observar, em outros tempos, que:

Para a maior parte das pessoas, constitui um enigma o fato de que eu possa trabalhar simultaneamente em disciplinas tão diferentes como Psicologia profunda, Sociologia, fisiologia, e agora também biologia. (...) O tema "sexualidade" atravessa realmente todos os campos científicos de pesquisa (Reich, 1984, p. 13). 
De qualquer forma, o registro dessa produção acadêmica visa a motivar o leitor a entrar em contato com as diferentes pesquisas brasileiras voltadas para o universo reichiano, as quais reforçam as inúmeras possibilidades de investigação científica calcadas nesse importante referencial.

Esperamos que, com posse desses dados, os pesquisadores interessados na investigação das produções acadêmicas na área reichiana possam aprofundar seus estudos, investigando, por exemplo, o conteúdo de cada uma dessas pesquisas. Em linhas gerais, é possível adiantar que, dentre as produções aqui registradas, existem aquelas que se concentram no aprofundamento de conceitos ou de temas específicos, enquanto outras aproximam o referencial reichiano do dia a dia profissional de diferentes áreas ou das ideias de autores consagrados, visando ao aprofundamento em questões contemporâneas de ordem política, social, educacional ou da saúde. O fato é que as produções acadêmicas que têm em Reich ou nas ideias reichianas seu objeto de estudo são cada vez mais frequentes no universo acadêmico brasileiro, ainda que possam ser consideradas muito restritas se comparadas a outros objetos de investigação mais tradicionais em termos de pesquisa. Entretanto, é preciso destacar que as pesquisas relacionadas a Reich tendem a aumentar, considerando, por exemplo, o número de docentes e pesquisadores titulados que adentram o universo acadêmico como professores de cursos de graduação e de pós-graduação os quais, certamente, multiplicarão as investigações nesse universo que merece ser amplamente conhecido.

Sara Quenzer Matthiesen

Doutora em Educação pela Unesp-Marília . Atualmente é docente da Universidade Estadual Paulista Júlio de Mesquita Filho, São Paulo - SP.

Email: saraqm@rc.unesp.br

Endereço para envio de correspondência:

Departamento de Educação Física da Unesp-Rio Claro, Av. 24A, n. 1515, Bairro Bela Vista, Rio Claro, São Paulo - SP. CEP 13506-807

Recebido 23/4/2010, Aprovado 15/10/2011. 
Matthiesen, S. Q. (2002). Caminho das pedras: as publicações de Wilhelm Reich em português. Rio Claro: Majograf.

Matthiesen, S. Q. (2005). A educação em Wilhelm Reich: da psicanálise à pedagogia econômico-sexual. São Paulo: Unesp.

Matthiesen, S. Q. (2007). Organização bibliográfica da obra de
Wilhelm Reich: base para o conhecimento em diferentes áreas do conhecimento. São Paulo: Annablume/Fapesp.

Reich, W. (1984). A função do orgasmo: problemas econômicosexuais da energia biológica (10a. ed.). São Paulo: Brasiliense. 